OPEN ACCESS

Edited by:

Leonardo Alexandre Peyré-Tartaruga, Universidade Federal do Rio Grande

do Sul (UFRGS), Brazil

Reviewed by:

Luiz Royes,

Universidade Federal de Santa Maria,

Brazil

Felipe P. Carpes,

Federal University of Pampa, Brazil

*Correspondence:

Walter Krause Neto

wild_krause@hotmail.com

Specialty section:

This article was submitted to

Integrative Physiology,

a section of the journal

Frontiers in Physiology

Received: 17 April 2018

Accepted: 12 June 2018

Published: 03 July 2018

Citation:

Pereira ES, Krause Neto W, Calefi AS, Georgetti M, Guerreiro L, Zocoler CAS and Gama EF (2018)

Significant Acute Response of Brain-Derived Neurotrophic Factor

Following a Session of Extreme

Conditioning Program Is Correlated With Volume of Specific Exercise

Training in Trained Men.

Front. Physiol. 9:823.

doi: 10.3389/fphys.2018.00823

\section{Significant Acute Response of Brain-Derived Neurotrophic Factor Following a Session of Extreme Conditioning Program Is Correlated With Volume of Specific Exercise Training in Trained Men}

\author{
Emy S. Pereira ${ }^{1,2}$, Walter Krause Neto ${ }^{1 *}$, Atilio S. Calefi ${ }^{3}$, Mariana Georgetti2, \\ Larissa Guerreiro'2, Cesar A. S. Zocoler ${ }^{4}$ and Eliane F. Gama ${ }^{1,2}$
}

'Laboratory of Morphoquantitative Studies and Immunohistochemistry, Department of Physical Education, São Judas Tadeu University, São Paulo, Brazil, ${ }^{2}$ Laboratory of Body Perception and Movement, Department of Physical Education, São Judas Tadeu University, São Paulo, Brazil, ${ }^{3}$ Department of Pathology, Faculty of Veterinary Medicine and Animal Science, University of São Paulo, São Paulo, Brazil, ${ }^{4}$ Laboratory of Human Movement, Department of Physical Education, São Judas Tadeu University, São Paulo, Brazil

Several studies have demonstrated an acute and chronic increase of brain-derived neurotrophic factor (BDNF) in relation to different types of physical exercise. Currently, many individuals seek physical training strategies that present different types of stimulation and volume/intensity. Thus, the extreme conditioning methodology has gained great notoriety in the scientific and non-scientific environment. Knowing that BDNF values increase in an effort-dependent manner, it is necessary to study the effects of this strategy on BDNF levels. This study aimed to evaluate the acute response of BDNF in trained men submitted to an extreme conditioning program (ECP) session. Ten volunteers underwent an acute ECP session using the "as many reps as possible" (WOD-AMRAP) method, including three types of exercise (clean, wall ball and double or single-unders) for 9 min. BDNF was measured in the plasma, being collected baseline and immediately after the session. Total load of the clean exercise was five times greater than wall ball exercise ( $p<0.05 ; 2096.1 \pm 387.4 \mathrm{~kg}$ vs $415.8 \pm 81.03 \mathrm{~kg}$ ), which influenced little in the total load $(p<0.05,2511.9 \pm 358.52 \mathrm{~kg})$ used. For the total volume, practitioners averaged 1.7 times more repetitions in the wall ball exercise compared to clean (46.2 \pm 9 vs $29.5 \pm 3.8$ repetitions). The volunteers averaged $75.7 \pm 12.6$ double-unders repetitions, bringing the total volume of training to $151.4 \pm 23.7$ repetitions. Regarding the BDNF values, there was a significant difference $(p=0.05)$ between the pre- vs post-moments (11209.85 \pm 1270.4 vs $12132.96 \pm 1441.93 \mathrm{pg} / \mathrm{ml})$. Effect size for this change as moderate $(E S=0.79)$. We found a positive correlation between total volume of clean exercise and delta BDNF values $(p=0.049)$. In conclusion, a single extreme conditioning session, through the 
practice of the WOD-AMRAP method, is capable of increasing the acute concentrations of plasma BDNF. In practical terms, we may suggest that future studies evaluate the effect of ECP as a strategy in the treatment of disorders associated with central degenerative changes.

Keywords: high-intensity interval training, effort, strength training, aerobics, cross training, neurotrophin

\section{INTRODUCTION}

Brain-derived neurotrophic factor (BDNF) is a potent neurotrophin found in many tissues, including hippocampus, skeletal muscle, cardiac muscle, and liver and adipose cells (Leibrock et al., 1989; Zafra et al., 1990; Yamamoto et al., 1996; Marosi and Mattson, 2014; Briana and Malamitsi-Puchner, 2017; Pius-Sadowska and Machaliński, 2017). In the central nervous system, this peptide stimulates neuroplasticity, through direct mediation of the formation of new neuronal circuits, neuronal survival and synaptogenesis (Gomez-Pinilla et al., 2008; Fargali et al., 2012; Numakawa et al., 2017). At the cellular level, BDNF acts via specific tyrosine kinase receptors, being directly involved in improving memory and learning in both experimental and human models (Falkenberg et al., 1992; Lapchak et al., 1993; von Bohlen Und Halbach and von Bohlen Und Halbach, 2018).

In peripheral tissues, as in skeletal muscle, BDNF appears to act in a paracrine rather than an endocrine manner (Matthews et al., 2009; Rasmussen et al., 2009; Maekawa et al., 2018). Its action, via receptor tyrosine kinase, stimulates local metabolism through enzymatic mechanisms activated by AMP-dependent kinase (Matthews et al., 2009).

Exercise and physical training seem to be potentially stimulating for the acute and chronic response of BDNF (Ferris et al., 2007; Seifert et al., 2010; Yarrow et al., 2010; Marquez et al., 2015; Liu and Nusslock, 2018). The majority of research investigating BDNF response to physical activity and exercise/physical training primarily examined aerobic exercise (Mackay et al., 2017; Liu and Nusslock, 2018). Acute increases in plasma BDNF seems to be related to effort duration and intensity (Ferris et al., 2007; Rojas et al., 2010; Seifert et al., 2010; Cho et al., 2012; Marquez et al., 2015). Literature data suggest that the higher volume, or its association with higher intensities, may influence the acute response of BDNF to aerobic exercise (Ferris et al., 2007; Cho et al., 2012).

Recent data suggest that resistance and/or concurrent training may also potentially stimulate plasma levels of neurotrophins (Cho et al., 2014; Church et al., 2016). However, an increasing number of individuals are seeking a type of exercise that mixes a greater diversity of stimuli within the same training session. Murawska-Cialowicz et al. (2015) demonstrated that extreme conditioning programs (ECPs) (such as CrossFit) altered BDNF levels at rest, after Wingate and treadmill testing, and improved aerobic capacity of active practitioners.

In the United States, ECP training emerged in the mid1980s, emphasizing the need to improve the physical fitness, becoming a very popular modality (Glassman, 2003; Murawska-Cialowicz et al., 2015). The ECP is a training method with a high level of effort/intensity during training sessions, in which its main goal is the improvement of the physical capacities (cardiorespiratory endurance, muscular resistance, strength, power, speed, coordination, flexibility, agility, balance, and precision). This training modality is based on Olympic weightlifting exercises (which are characterized by generating greater muscular power and the need for motor coordination), gymnastic movements (body weight or calisthenic exercises) and metabolic exercises (emphasized with cyclical movements such as running, swimming, cycling, single/double-unders and others). Also, ECP training centers is known to work with great amount of repetitions (volume), high intensity (load), high density (small periods of interval, or still absent) and small periods of stimulation (minutes), characterizing a high level of effort. Knowing that high-level effort (whether by volume or intensity) may affect the acute response of BDNF (Church et al., 2016), it is intriguing to investigate the acute response of this neurotrophin to ECPs and the influence of training parameters on BDNF changes.

According to Glassman (2010), ECP contributes to the overall development of an individual's body and mind performance. Recently, Murawska-Cialowicz et al. (2015) demonstrated that 3 months of ECP training increased rest levels of BDNF. However, the study was performed using physically active individuals, but not previously trained in this modality. In addition, the acute parameters of BDNF were measured only using cyclic tests (Wingate and maximum progressive in cycle ergometer) and not specific ECP exercises or routine. Thus, based on their results, it is not possible to conclude that the short, voluminous and intense ECP sessions are efficient in stimulating a significant acute increase of BDNF in already trained individuals. Nevertheless, a more complete picture of the responses of this neurotrophin may indicate ECP as possible training protocol for people who are deficient in the action of this protein.

Knowing the need to understand the acute response to the specific training session of the modality, this study aimed to evaluate the acute response of BDNF in individuals trained in ECP undergoing a "workout of the day" (WOD) session. In addition, we investigated which training parameter (such as volume, load or specific exercise component) influenced the acute response of this neurotrophin.

\section{MATERIALS AND METHODS}

This study was approved by the Ethics Committee of the São Judas Tadeu University under number 37330614.7.0000.0089, in accordance with Resolution 466/12 of the National Health 
Council, which regulates the methodological procedures in research with human beings.

\section{Volunteers}

Adult males who had been training ECP for at least 6 months (Table 1) were selected for this study (Dinoff et al., 2017). Initially, 12 individuals applied to participate in the research. After analyzing the profiles according to the inclusion and exclusion criteria, 10 were selected for the training session. This sample size is sufficient to answer our research question, as has already been demonstrated in the literature (Seifert et al., 2010; MurawskaCialowicz et al., 2015; Church et al., 2016). Participants who were in two or more concurrent training programs and/or under the use of anabolic steroids, psychotropic drugs, antibiotics and corticosteroids, and injured were excluded.

All volunteers were evaluated (blood collection) at two different times: baseline (just before start the training session) and immediately after the end of the training session (Figure 1).

\section{Training Protocol}

All evaluations were performed during the afternoon and each subject was submitted to the training protocol alone, in order to avoid the lack of attention to the training session and external influences (each subject was verbally stimulated only by the evaluators). All analysis was performed on 2 days, separated by $48 \mathrm{~h}$, and volunteers were asked not to do any strenuous exercise for at least $72 \mathrm{~h}$ prior testing.

On day 1, the term of free and informed consent was read together with the participants and all doubts were clarified. Also, the consent for both the study participation and publication of the images was both written and informed for all the participants (Figures 2, 3). After reading and agreeing to participate in the survey, individuals respond to the International Physical Activity Questionnaire (IPAQ), the Physical Activity Readiness Questionnaire (PAR-Q) and general anamnesis. These questionnaires showed us that volunteers had the level of physical activity required in this study and that all were free of any physical limitation. Afterward, all volunteers performed a load test (RM)
TABLE 1 | Descriptive data of the sample.

\begin{tabular}{lc}
\hline Data & Characteristics \\
\hline Subjects $\left(\mathrm{n}^{\circ}.\right)$ & 10 \\
Age (years) & $31 \pm 5$ \\
Height $(\mathrm{m})$ & $1.75 \pm 0.04$ \\
Weight $(\mathrm{kg})$ & $83.9 \pm 3.72$ \\
Training experience (months) & $22 \pm 9$ \\
\hline
\end{tabular}

for clean exercise (as protocol described by Ploutz-Snyder and Giamis, 2001). Thus, the volunteers performed two sets of 510 repetitions, with pauses of $2-3 \mathrm{~min}$. Shortly thereafter, the volunteers had up to five attempts, separated by 3-5 $\mathrm{min}$, to lift the maximum load in a single movement. From this moment, we calculate the load equivalent to $80 \%$ RM for the training session. Finally, we clarify any doubts of the volunteers regarding the execution of the exercises wall ball and double or single-unders.

On day 2, thirty minutes after the baseline blood collection, all volunteers were submitted to general body warming exercises composed of 3 sets of 10 jump air squat and kettlebell swing movements (Figure 2). This warm-up routine was chosen by specifically preparing the requested muscle chains to follow. The training was composed of three fundamental elements in the practice of ECP (Figure 3): weightlifting exercise (clean), gymnastic exercise (wall ball throw) and metabolic and/or cyclic exercise (double-unders and/or single-unders).

The training session was composed of the following: workout of the day - as many repetitions as possible (method WODAMRAP) in 9 min: 5 repetitions of cleans, 10 repetitions of wall ball throw, and 20 repetitions of double-unders or single-unders. After each successful sets of exercises, an extra movement was added (except for double-unders and/or single-unders), making the training session more strenuous (e.g., first series 5 cleans, 10 wall ball, 20 double-unders, and/or single-unders; second series 6 cleans, 11 wall ball, 20 double-unders, and/or singleunders; and so on until the completion of time). No rest breaks were allowed during session time. The training load of the clean

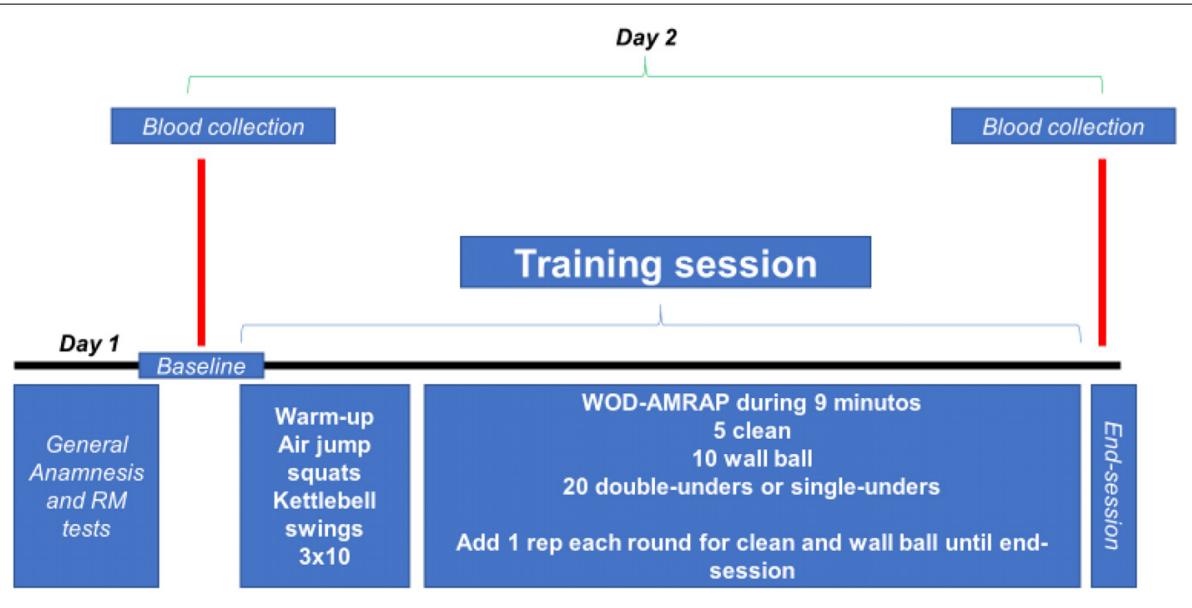

FIGURE 1 | Experimental design. WOD-AMRAP: workout of the day - as many reps as possible. 


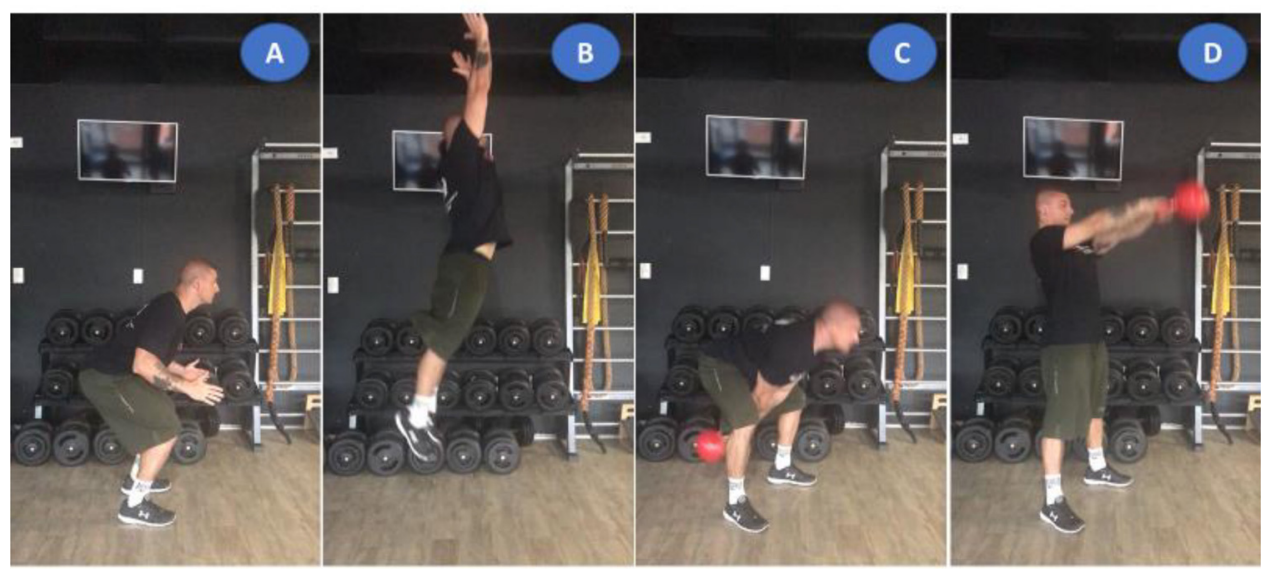

FIGURE 2 | |llustration of general body warming composed of three jump air squat (A,B) and kettlebell swing (C,D) movements. The consent for both the study participation and publication of the images was both written and informed for all the participants.

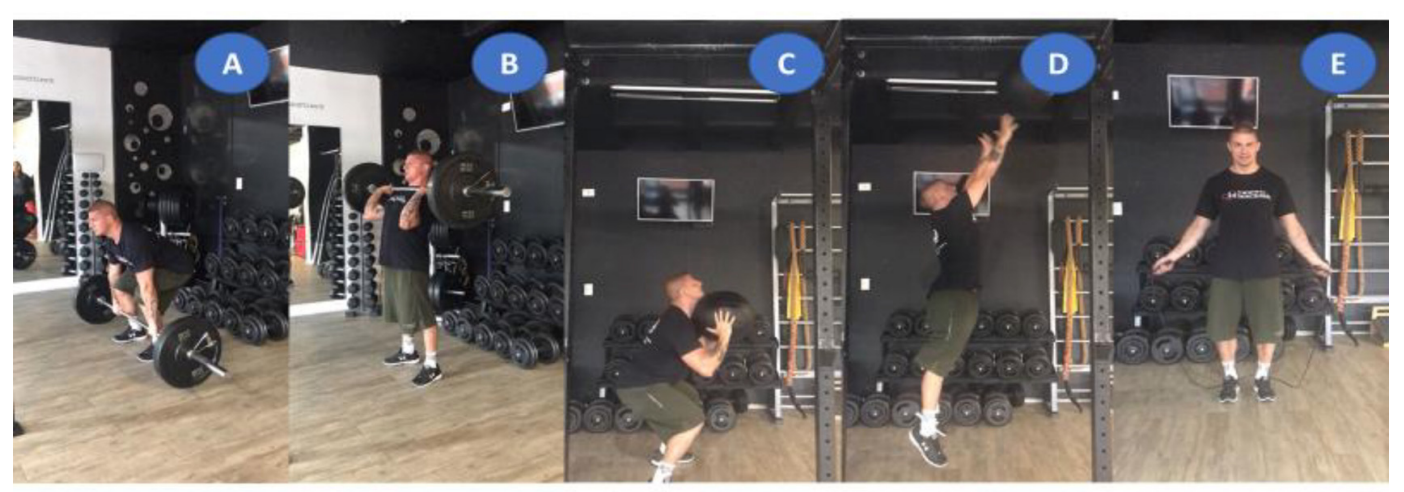

FIGURE 3 | Illustration of the extreme conditioning program training session composed of clean (A,B), wall ball (C,D), and double-unders (E) exercises. Informed consent was informed, written and obtained from this volunteer for publication of their images.

exercise was $80 \%$ RM (estimated in previous session) and for wall ball throw $9 \mathrm{~kg}$ (common load used by trained individuals for this exercise). Figure 1 presents experimental design of this study.

To quantify the total volume (TV) and total load volume (TVL), we used the following procedures: (1) TV, we sum the total number of repetitions of the clean and wall ball exercises; and (2) TVL, the TV was multiplied by the total load of each exercise. For the calculation of TV of the double-unders exercise, the total number of repetitions performed over time of WODAMRAP was added. Finally, we added the volume reps of all three exercises as total training volume (TTV).

\section{Blood Collection}

Blood samples were collected baseline (before warm-up exercise) and immediately after the training session. During the collection, the volunteers remained seated and right arm resting on a table. Approximately $10 \mathrm{ml}$ of venous blood was collected in a vacuum-disposed plastic tube with no additive. After collection, the tubes were left for $30 \mathrm{~min}$ at room temperature and subsequently held in a styrofoam box with ice.

After the blood collection, the samples were processed in the Laboratory of Biochemistry of the São Judas Tadeu University, being centrifuged at 5,000 rpm for $15 \mathrm{~min}$. After centrifugation, only the supernatant, consisting of $500 \mu 1$ Eppendorf conical tube serum, was separated with identification of the individuals at each time point.

All these procedures were carried out in a clean and sterilized workbench, using the appropriate protection precautions. The samples were then stored in a freezer at $-80^{\circ} \mathrm{C}$.

After obtaining the biological material from all individuals, the blood samples were sent to the Laboratory of Pharmacology and Toxicology of the Faculty of Veterinary Medicine and Animal Science of the University of São Paulo, in which the serum concentrations of BDNF were measured.

Serum BDNF concentrations were measured by enzymelinked immunosorbent assay (ELISA) technique. The technique relies on the use of antigens or antibodies labeled with an enzyme, so that the resulting conjugates have both immunological 
and enzymatic activity. It has one of the components (antigen or antibody) fixed on an adsorbent support, the antigen conjugate complex is immobilized, and the reaction can be easily revealed by the addition of a specific substrate that can act with the enzyme producing a color visible to the naked eye or quantifiable through the use of colorimetric and spectrophotometric techniques. The DuoSet $^{\circledR}$ Human BDNF kit (R\&D Systems, Minneapolis, MN, United States) was used. The kit was prepared according to the manufacturer's standards and instructions, and final results were expressed in $\mathrm{pg} / \mathrm{ml}$.

\section{Statistical Analysis}

All results were presented as mean \pm standard deviation. To test the normality of the data we used the ShapiroWilk test. To compare pre vs post-moments, Student's $t$-test was performed. One-way ANOVA was used to compare the TV and TVL outcomes (post hoc Tukey) between exercises. For the correlation tests between TV, TVL, and the BDNF delta values, Pearson's correlation test was used. The Cohen's $d$ effect size calculation (ES $=$ difference between pre- and post-intervention divided by pre-intervention SD) was used to evaluate the magnitude of BDNF changes. ES values were determined from very small (0.01-0.19), small (0.20$0.49)$, moderate $(0.50-0.79)$, large $(0.80-1.19)$, and very large $(1.20<)$. For the calculation of the data we used SPSS software version 21.0 and the level of significance was $p \leq 0.05$.

\section{RESULTS}

The TVL of the clean exercise was five times greater than wall ball exercise $(p<0.05 ; 2096.1 \pm 387.4 \mathrm{~kg}$ vs $415.8 \pm 81.03 \mathrm{~kg})$, which influenced little in the total load $(p<0.05,2511.9 \pm 358.52 \mathrm{~kg})$ used in WOD-AMRAP (Figure 4).

For the TV, practitioners averaged 1.7 times more repetitions in the wall ball compared to clean exercise ( $46.2 \pm 9$ vs $29.5 \pm 3.8$ repetitions). Total volume (adding both exercise repetitions)

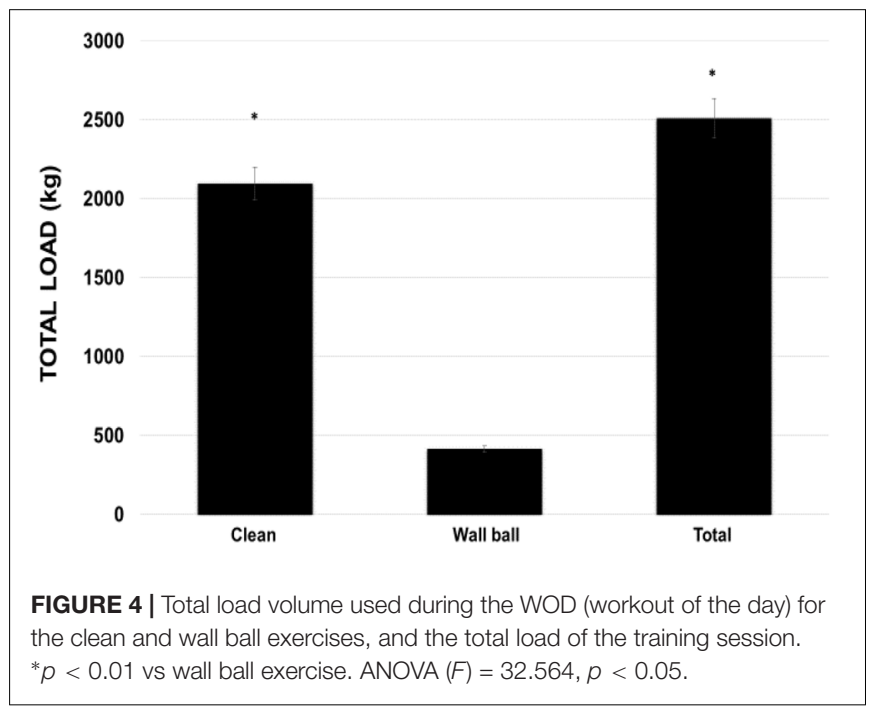

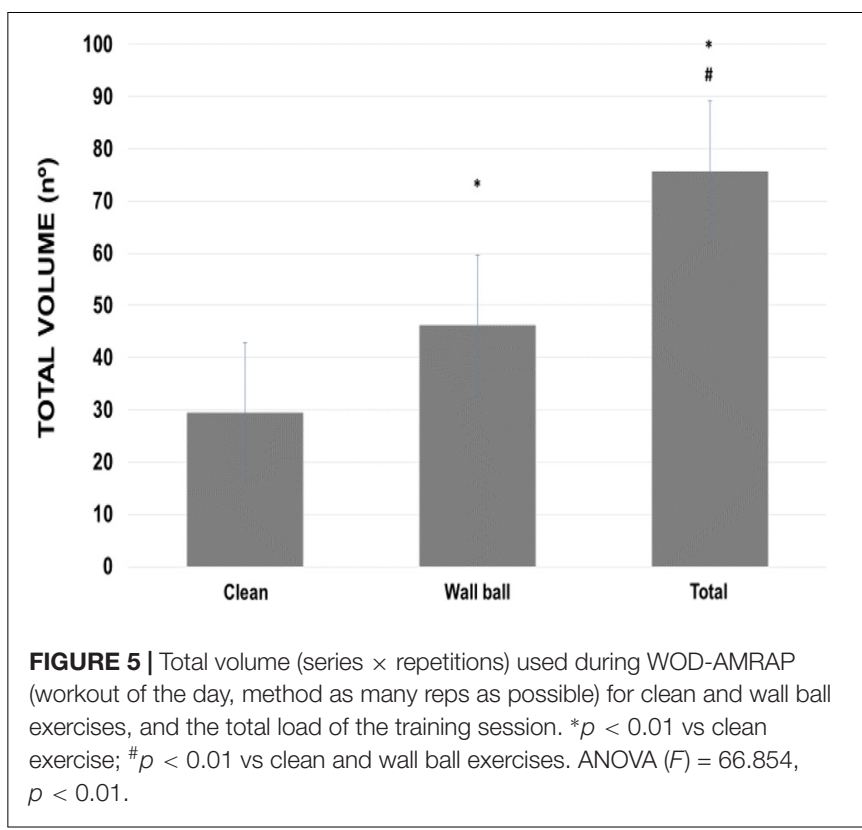

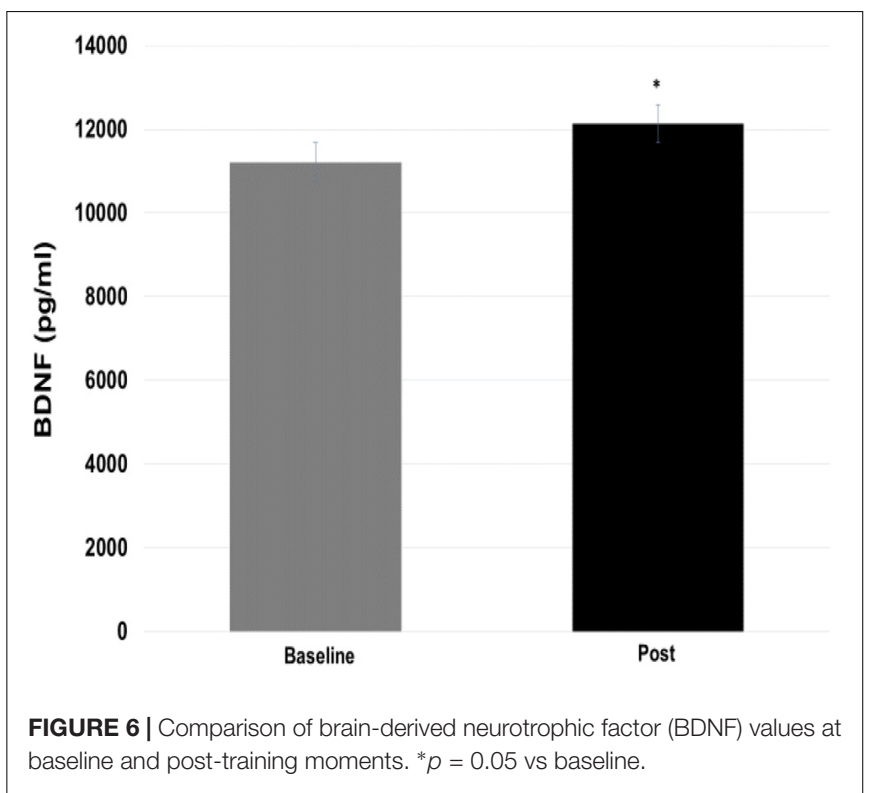

(75.7 \pm 12.2 repetitions) was statistically higher $(p<0.01)$ than each exercise analyzed alone (Figure 5). The volunteers averaged $75.7 \pm 12.6$ double-unders repetitions, bringing the TTV to $151.4 \pm 23.7$ repetitions.

Regarding the BDNF values (Figure 6), there was significant difference $(p=0.05)$ between the pre- vs post-moments $(11209.85 \pm 1270.4$ vs $12132.96 \pm 1441.93 \mathrm{pg} / \mathrm{ml})$. The effect size for this change was shown to be moderate $(\mathrm{ES}=0.73)$.

Table 2 shows the correlation values between the training parameters (total volume and total load) and BDNF delta value changes. Figure 7 represents a positive correlation between total volume of clean exercise and delta BDNF values $(p=0.049)$. For other comparisons, there was no significant correlation. 
TABLE 2 | Correlation between total volume (series $\times$ replicates) and total load (total volume $\times$ weight) of training parameters with values of delta brain-derived neurotrophic factor (BDNF) changes.

\begin{tabular}{ll}
\hline & BDNF (delta) \\
\hline Total clean load $(\mathrm{kg})$ & 0.184 \\
Total clean reps $\left(\mathrm{n}^{\circ}.\right)$ & $0.633^{*}$ \\
Total wall ball load $(\mathrm{kg})$ & 0.259 \\
Total wall ball reps $\left(\mathrm{n}^{\circ}.\right)$ & 0.259 \\
Total reps double unders $\left(\mathrm{n}^{\circ}.\right)$ & 0.588 \\
Total load $(\mathrm{kg})$ & 0.257 \\
Total reps clean + wall ball $\left(\mathrm{n}^{\circ}.\right)$ & 0.387 \\
Total reps clean + wall ball + double unders $\left(\mathrm{n}^{\circ}.\right)$ & 0.512
\end{tabular}

Asterisk "*" indicates significant correlation $p<0.05$.

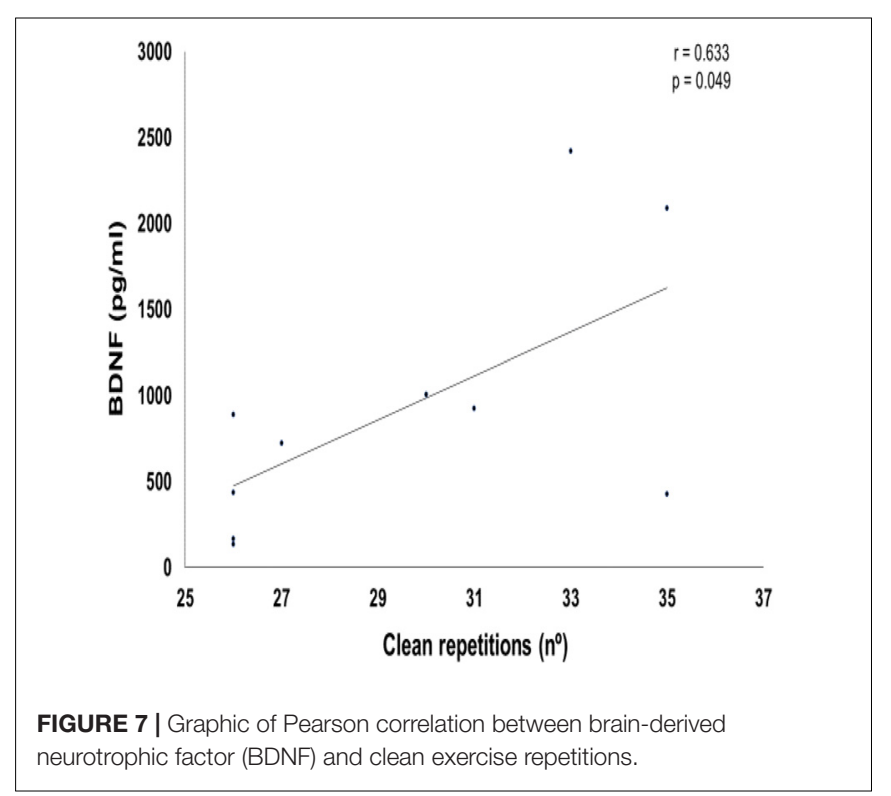

\section{DISCUSSION}

Here, we demonstrate two new facts: (1) as other types of exercise, a single session of ECP is significantly potent to stimulate acute increase of plasma BDNF levels, and (2) the number of repetitions of the clean exercise was positively correlated with this physiological effect.

Our results suggest that the combination of multiple types of exercise, high effort, high intensity, and high training volume in a relatively short time $(9 \mathrm{~min})$ can be a decisive factor in the dynamics of post-exercise changes in BDNF. Here, we confirmed results already found by other research groups, whose purpose was to investigate the effects of more common types of exercises on alterations of this neurotrophin (Seifert et al., 2010; Szuhany et al., 2015; Park and Kwak, 2017; Liu and Nusslock, 2018).

Generally, literature studies give more attention to the influence of aerobic exercise on BDNF secretion compared to predominantly anaerobic or strength exercise. Zoladz et al. (2008) observed an increase in acute levels of BDNF after a single aerobic exercise session. This phenomenon has being found in several different populations (Mackay et al., 2017;
Hsueh et al., 2018; Morais et al., 2018). However, the results of our study do not seem to be influenced by the doubleunders exercise (aerobic type exercise), as presented through the correlation tests. Therefore, the influence of multi-articular strength exercises on the BDNF response was fundamental. In isolation, the results are controversial as to the efficiency of strength training in increasing the plasma levels of this neurotrophin (Rojas Vega et al., 2006; Goekint et al., 2010; Yarrow et al., 2010). Possibly, the circuit training characteristic used here, was fundamental to our result. Associating the specific characteristics of the three exercises included in our study, with the high volume-intensity methodology, we demonstrated a potent effect of ECP training on BDNF levels. In general, the literature should better explore various types of program assembly in order to give practical possibilities of the results already demonstrated.

Pearson's correlation positively pointed to the influence of the number of repetitions performed in the clean exercise on the BDNF delta. Thus, the type of exercise, the percentage of the maximum load used and the total volume of exercise training involving large muscle mass, may influence the acute responses of this neurotrophin. In accordance with our results, Lee and Soya (2017) demonstrated that acute loaded wheel running increased hippocampal BDNF activity in rats. In addition, Jeon and Ha (2017) demonstrated that moderate to high effort levels of aerobic training seems to have a positive effect on levels of BDNF at rest and on cognitive function. For the other side, Church et al. (2016) indicated that BDNF levels are increased after an acute session of resistance exercise, regardless of training differences protocols. These data confirms the need for more studies regarding the influence of different training variables on the BDNF acute responses.

Most of the exercises used here, involved moderate to high external load and could be classified as a predominantly resisted activity. Still, individuals should perform as many repetitions as possible within a predetermined time. Thus, the levels of fatigue achieved were very close to the maximum for each individual. Marston et al. (2017) used a method of training to-fatigue based resistance exercise protocol and quoted that this activity provides the necessary stimulus to increase peripheral serum BDNF. Perhaps, strenuous exercise or higher total volume may also have some influence on the outcomes measured here. Cho et al. (2014) demonstrated that although there was no difference in serum BDNF levels between aerobic and combined type exercises, higher stimulus may have a possibility of positive changes in increase of serum BDNF level in mid-aged women. Cabral-Santos et al. (2016) indicated that even different volume training would not change acute BDNF values if aerobic exercise intensity are maintained. Being so, Forti et al. (2015) showed that only a very high volume at a sufficiently high external load was able to increase circulating BDNF. These data corroborates our findings that fast and powerful movements with high intensity ( $80 \%$ maximum load) and higher number of repetitions can be enough to stimulate the increase in BDNF levels. Also, the amount of muscle mass used might also have an impact in the neurotrophin levels. 
Walsh et al. (2016) demonstrated a transient increase in serum BDNF following a single session of strength exercise in older adults after lower body resistance training. Therefore, training to voluntary fatigue might be necessary to obtain significant BDNF levels increase.

The exercises used in ECP involve the whole body, unlike those exercised in a conventional gym. Here, we include different types of exercises, such as power (clean), strength resistance (wall ball throwing) and aerobic (double-unders) exercises. This variety of exercise stimuli can activate different physiological mechanisms. According to Cassilhas et al. (2012), both exercise types (aerobic and strength) present distinct signaling pathways. Aerobic type exercise presents an increased level of IGF-1, BDNF, TrkB, and $\beta$-CaMKII (calcium/calmodulin-dependent kinase II) in the hippocampus, whereas the strength type of training showed an induction of peripheral and hippocampal IGF-1 with concomitant activation of receptor for IGF-1 (IGF1R) and Akt in the hippocampus. These cellular pathways stimulated synapsin 1 and synaptophysin expression in both groups. These data confirmed that aerobic and strength exercise can stimulated different molecular mechanisms with similar results on learning and spatial memory. These data suggest that individuals undergoing a session, or training, using methods with various types of exercise could benefit in both signaling pathways.

Our training strategy can be effective in improving memory and learning, as seen in the prescription of more traditional exercises. In terms of applicability, this methodology can be classified as time-efficient. Thus, high-level stress sessions can be done in high training density, making it interesting as a non-medicament treatment in several degenerative dysfunctions. Tharmaratnam et al. (2018) demonstrated that 12 weeks of moderate-to-high intensity training is able to improve working memory by increasing brain oxygenation, nutrient delivery and BDNF mRNA expression in adolescents. However, higher training intensity demonstrates larger and more significant results on working memory and increase of BDNF compared to lower intensities (Jeon and Ha, 2017). Etnier et al. (2016) showed that long-term memory differed as a function of exercise intensity with better results being presented after maximal intensity levels, although BDNF increase after all exercise intensities. Pietrelli et al. (2018) presented data showing that exercise improves memory and learning thru the system serotoninBDNF, protecting the brain against deleterious effects. These data demonstrate that both acute and chronic changes are associated with improved memory, learning and cognition, and are corroborated by other studies in several other conditions (Hopkins and Bucci, 2010; Hopkins et al., 2011; Håkansson et al., 2017). However, the large amount of data present in the literature was investigated using continuous aerobic exercise, thus requiring more investigations confirming the same effect in consideration of other types of methodologies and exercises.

Mechanisms that altered resting BDNF levels in bloodstream can be different. During exercise with high intensity or volume (as used here), lactate plasma levels are frequently increased due to the high mobilization of glycolytic metabolism. Schiffer et al.
(2011) demonstrated a significant increase in BDNF levels while administering sodium lactate adult individuals. The authors quoted that lactate might participate in the regulation of BDNF production levels. However, according to Marston et al. (2017), the presence of lactate does not appear to stimulate the BDNF response during resistance exercise. Clearly, there is the need of further studies on this subject.

Further, exhaustive training sessions may stimulate increased expression and protein production of oxidative stress. Sakr et al. (2015) observed a significant augment in the levels of cortical malondialdehyde, a reduction in antioxidant activity (decreased glutathione, superoxide dismutase, and catalase) and a significant increase in BDNF expression in rats subjected to chronic effort under hypoxic conditions. These data presents evidence that oxidative stress could be an important factor stimulating BDNF expression. For the first time, Freitas et al. (2018) demonstrated a significant effect of HIIT on reducing oxidative stress, lipoperoxidation and inflammatory markers, as well enhancing antioxidant defenses and BDNF content.

In ECP sessions, adrenergic levels rise, modulating the energy response, and may stimulate feelings of well-being in the post-session. Chen and Russo-Neustadt (2007) demonstrated that norepinephrine and serotonin are responsible for inducing BDNF expression. Norepinephrine induces phosphorylation of cAMP-response element binding protein (CREB) and stimulates expression of both BDNF and nerve growth factor (NGF) (Counts and Mufson, 2010). Also, norepinephrine activates phosphatidylinositol-3 kinase (PI-3K)/Akt and mitogen-activated protein kinase (MAPK) and NO/cGMP pathways, in hippocampal neurons (Chen and Russo-Neustadt, 2013). BDNF activates the Trk receptor and participate in neuron plasticity, growth, development and survival of neurons.

Finally, the realization of ECP sessions requires high energy demand. It is fundamental to understand the action of BDNF in central and peripheral tissues. Wrann et al. (2013) link endurance exercise and the important metabolic mediators, PGC- $1 \alpha$ and FNDC5, with BDNF expression in the brain. FNDC5, a muscle protein cleaved and secreted as irisin, is also elevated by endurance exercise in the hippocampus of rodents. Further, neuronal Fndc5 gene expression is regulated by PGC$1 \alpha$, and PGC-1 $\alpha$ knockout mice show reduced Fndc5 expression in the brain. Importantly, peripheral delivery of FNDC5 to the liver via adenoviral vectors, resulting in elevated blood irisin, induces expression of $B d n f$ and other neuroprotective genes in the hippocampus (Wrann et al., 2013). The findings from this work indicate that endurance exercise increase the activity of the ERR $\alpha /$ PGC- $1 \alpha$ proteins, which increases levels of FNDC5 in skeletal muscle and hippocampus (Xu, 2013). Murawska-Cialowicz et al. (2015) demonstrated that 3 months of ECP training was able to increase resting BDNF levels without any alterations in irisin plasmatic concentration. This data may show that irisin may act as a paracrine regulator of BDNF.

Based on the results found here, we can conclude that a single ECP session, through the practice of the WOD-AMRAP method, 
is capable of increasing the acute concentrations of plasma BDNF. Secondly, we can also conclude that the total volume of repetitions of the clean Olympic exercise, when performed with high intensity ( $80 \% \mathrm{RM})$, correlates positively with the increase of BDNF found.

\section{AUTHOR CONTRIBUTIONS}

EP is the lead author of this manuscript, contributing all of its steps including volunteer training. WKN assisted in the steps of project preparation, statistics, data analysis, and final data preparation. AC collected the data and blood, and analyzed the BDNF. MG, LG, and CZ assisted in the assembly of the training protocol, data collection during training of the volunteers, and in the final version of the

\section{REFERENCES}

Briana, D. D., and Malamitsi-Puchner, A. (2017). Developmental origins of adult health and disease: the metabolic role of BDNF from early life to adulthood. Metabolism 81, 45-51. doi: 10.1016/j.metabol.2017.11.019

Cabral-Santos, C., Castrillón, C. I., Miranda, R. A., Monteiro, P. A., Inoue, D. S., Campos, E. Z., et al. (2016). Inflammatory cytokines and BDNF response to high-intensity intermittent exercise: effect the exercise volume. Front. Physiol. 7:509. doi: 10.3389/fphys.2016.00509

Cassilhas, R. C., Lee, K. S., Fernandes, J., Oliveira, M. G., Tufik, S., Meeusen, R., et al. (2012). Spatial memory is improved by aerobic and resistance exercise through divergent molecular mechanisms. Neuroscience 202, 309-317. doi: 10. 1016/j.neuroscience.2011.11.029

Chen, M., and Russo-Neustadt, A. (2013). Kinetics of norepinephrine- and serotonin-induced BDNF release in cultured embryonic hippocampal neurons. Neurosci. Med. 4, 194-207. doi: 10.4236/nm.2013.44031

Chen, M. J., and Russo-Neustadt, A. A. (2007). Nitric oxide signaling participates in norepinephrine-induced activity of neuronal intracellular survival pathways. Life Sci. 81, 280-290. doi: 10.1016/j.lfs.2007.09.003

Cho, H., Kim, J., Kim, S., Son, Y. H., Lee, N., and Jung, S. H. (2012). The concentrations of serum, plasma and platelet BDNF are all increased by treadmill VO2max performance in healthy college men. Neurosci. Lett. 519, 78-83. doi: 10.1016/j.neulet.2012.05.025

Cho, H. C., Kim, J. K., Lee, N. J., Kim, S. Y., and Yoon, N. K. (2014). Effects of combined exercise on cardiovascular risk factors and serum BDNF level in mid-aged women. J. Exerc. Nutr. Biochem. 18, 61-67. doi: 10.5717/jenb.2014. 18.1.61

Church, D. D., Hoffman, J. R., Mangine, G. T., Jajtner, A. R., Townsend, J. R., Beyer, K. S., et al. (2016). Comparison of high-intensity vs. high-volume resistance training on the BDNF response to exercise. J Appl Physiol 121, 123-128. doi: 10.1152/japplphysiol.00233.2016

Counts, S., and Mufson, E. J. (2010). Noradrenaline activation of neurotrophic pathways protects against neuronal amyloid toxicity. J. Neurochem. 113, 649-660. doi: 10.1111/j.1471-4159.2010.06622.x

Dinoff, A., Herrmann, N., Swardfager, W., and Lanctôt, K. L. (2017). The effect of acute exercise on blood concentrations of brain-derived neurotrophic factor in healthy adults: a meta-analysis. Eur. J. Neurosci. 46, 1635-1646. doi: 10.1111/ ejn. 13603

Etnier, J. L., Wideman, L., Labban, J. D., Piepmeier, A. T., Pendleton, D. M., Dvorak, K. K., et al. (2016). The effects of acute exercise on memory and brain-derived neurotrophic factor (BDNF). J. Sport Exerc. Psychol. 38, 331-340. doi: 10.1123/jsep.2015-0335

Falkenberg, T., Mohammed, A. K., Henriksson, B., Persson, H., Winblad, B., and Lindefors, N. (1992). Increased expression of brain-derived neurotrophic factor mRNA in rat hippocampus is associated with improved spatial memory and enriched environment. Neurosci. Lett. 138, 153-156. doi: 10.1016/03043940(92)90494-R manuscript. EG guided the entire process and was present at all stages.

\section{FUNDING}

We appreciate the support received from FAPESP, which provided funds for the publication of this article (Case No. 2018/13456-6, São Paulo State Research Support Foundation FAPESP).

\section{ACKNOWLEDGMENTS}

We would like to thank all those who have somehow made this work possible.

Fargali, S., Sadahiro, M., Jiang, C., Frick, A. L., Indall, T., Cogliani, V., et al. (2012). Role of neurotrophins in the development and function of neural circuits that regulate energy homeostasis. J. Mol. Neurosci. 48, 654-659. doi: 10.1007/s12031-012-9790-9

Ferris, L. T., Williams, J. S., and Shen, C. L. (2007). The effect of acute exercise on sérum brain-derived neurotrophic factor levels and cognitive function. Med. Sci. Sports Exerc. 39, 728-734. doi: 10.1249/mss.0b013e31802f04c7

Forti, L. N., Van Roie, E., Njemini, R., Coudyzer, W., Beyer, I., Delecluse, C., et al. (2015). Dose-and gender-specific effects of resistance training on circulating levels of brain-derived neurotrophic factor (BDNF) in communitydwelling older adults. Exp. Gerontol. 70, 144-149. doi: 10.1016/j.exger.2015. 08.004

Freitas, D. A., Rocha-Vieira, E., Soares, B. A., Nonato, L. F., Fonseca, S. R., Martins, J. B., et al. (2018). High-intensity interval training modulates hippocampal oxidative stress, BDNF and inflammatory mediators in rats. Physiol. Behav. 184, 6-11. doi: 10.1016/j.physbeh.2017.10.027

Glassman, G. (2003). Metabolic conditioning. CrossFit J. 1-2.

Glassman, G. (2010). Seminars Training Guide. CrossFit Level 1 Training Guide. Available at: http://library.crossfit.com/free/pdf/CFJ_English_Level1_ TrainingGuide.pdf

Goekint, M., De Pauw, K., Roelands, B., Njemini, R., Bautmans, I., Mets, T., et al. (2010). Strength training does not influence serum brain-derived neurotrophic factor. Eur. J. Appl. Physiol. 110, 285-293. doi: 10.1007/s00421-010-1461-3

Gomez-Pinilla, F., Vaynman, S., and Ying, Z. (2008). Brain-derived neurotrophic factor functions as a metabotrophin to mediate the effects of exercise on cognition. Eur. J. Neurosci. 28, 2278-2287. doi: 10.1111/j.1460-9568.2008. 06524.x

Håkansson, K., Ledreux, A., Daffner, K., Terjestam, Y., Bergman, P., Carlsson, R., et al. (2017). BDNF responses in healthy older persons to 35 minutes of physical exercise, cognitive training, and mindfulness: associations with working memory function. J. Alzheimers Dis. 55, 645-657. doi: 10.3233/JAD160593

Hopkins, M. E., and Bucci, D. J. (2010). BDNF expression in perirhinal cortex is associated with exercise-induced improvement in object recognition memory. Neurobiol. Learn. Mem. 94, 278-284. doi: 10.1016/j.nlm.2010. 06.006

Hopkins, M. E., Nitecki, R., and Bucci, D. J. (2011). Physical exercise during adolescence versus adulthood: differential effects on object recognition memory and brain-derived neurotrophic factor levels. Neuroscience 194, 84-94. doi: 10.1016/j.neuroscience.2011.07.071

Hsueh, S. C., Chen, K. Y., Lai, J. H., Wu, C. C., Yu, Y. W., Luo, Y., et al. (2018). Voluntary physical exercise improves subsequent motor and cognitive impairments in a rat model of Parkinson's disease. Int. J. Mol. Sci. 19, E508. doi: 10.3390/ijms19020508

Jeon, Y. K., and Ha, C. H. (2017). The effect of exercise intensity on brain-derived neurotrophic factor and memory in adolescents. Environ. Health Prev. Med. 22:27. doi: 10.1186/s12199-017-0643-6 
Lapchak, P. A., Araujo, D. M., Beck, K. D., Finch, C. E., Johnson, S. A., and Hefti, F. (1993). BDNF and trkB mRNA expression in the hippocampal formation of aging rats. Neurobiol. Aging 14, 121-126. doi: 10.1016/0197-4580(93)90087- R

Lee, M., and Soya, H. (2017). Effects of acute voluntary loaded wheel running on BDNF expression in the rat hippocampus. J. Exerc. Nutr. Biochem. 21, 52-57. doi: $10.20463 /$ jenb.2017.0034

Leibrock, J., Lottspeich, F., Hohn, A., Hofer, M., Hengerer, B., Masiakowski, P., et al. (1989). Molecular cloning and expression of brain-derived neurotrophic factor. Nature 341, 149-152. doi: 10.1038/341149a0

Liu, P. Z., and Nusslock, R. (2018). Exercise-mediated neurogenesis in the hippocampus via BDNF. Front. Neurosci. 12:52. doi: 10.3389/fnins.2018. 00052

Mackay, C. P., Kuys, S. S., and Brauer, S. G. (2017). The effect of aerobic exercise on brain-derived neurotrophic factor in people with neurological disorders: a systematic review and meta-analysis. Neural Plast. 2017:4716197. doi: 10.1155/ $2017 / 4716197$

Maekawa, T., Ogasawara, R., Tsutaki, A., Lee, K., Nakada, S., Nakazato, K., et al. (2018). Electrically evoked local muscle contractions cause an increase in hippocampal BDNF. Appl. Physiol. Nutr. Metab. 43, 491-496. doi: 10.1139/ apnm-2017-0536

Marosi, K., and Mattson, M. P. (2014). BDNF mediates adaptive brain and body responses to energetic challenges. Trends Endocrinol. Metab. 25, 89-98. doi: 10.1016/j.tem.2013.10.006

Marquez, C. M. S., Vanaudenaerde, B., Troosters, T., and Wenderoth, N. (2015). High-intensity interval training evokes larger serum BDNF levels compared to intense continuous exercise. J. Appl. Physiol. 119, 1363-1371. doi: 10.1152/ japplphysiol.00126.2015

Marston, K. J., Newton, M. J., Brown, B. M., Rainey-Smith, S. R., Bird, S., Martins, R. N., et al. (2017). Intense resistance exercise increases peripheral brain-derived neurotrophic factor. J. Sci. Med. Sport 20, 899-903. doi: 10.1016/j.jsams.2017. 03.015

Matthews, V. B., Aström, M. B., Chan, M. H. S., Bruce, C. R., Krabbe, K. S., Prelovsek, O., et al. (2009). Brain-derived neurotrophic factor is produced by skeletal muscle cells in response to contraction and enhances fat oxidation via activation of AMP-activated protein kinase. Diabetologia 52, 1409-1418. doi: $10.1007 / \mathrm{s} 00125-009-1364-1$

Morais, V. A. C., Tourino, M. F. D. S., Almeida, A. C. S., Albuquerque, T. B. D., Linhares, R. C., Christo, P. P., et al. (2018). A single session of moderate intensity walking increases brain-derived neurotrophic factor (BDNF) in the chronic post-stroke patients. Top. Stroke Rehabil. 25, 1-5. doi: 10.1080/ 10749357.2017.1373500

Murawska-Cialowicz, E., Wojna, J., and Zuwala-Jagiello, J. (2015). Crossfit training changes brain-derived neurotrophic factor and irisin levels at rest, after Wingate and progressive tests, and improves aerobic capacity and body composition of Young physically active men and women. J. Physiol. Pharmacol. 66, 811-821.

Numakawa, T., Odaka, H., and Adachi, N. (2017). Actions of brain-derived neurotrophic factor and glucocorticoid stress in neurogenesis. Int. J. Mol. Sci. 18:E2312. doi: 10.3390/ijms18112312

Park, C. H., and Kwak, Y. S. (2017). Analysis of energy restriction and physical activity on brain function: the role of ketone body and brain-derived neurotrophic factor. J. Exerc. Rehabil. 13, 378-380. doi: 10.12965/jer.1735028. 514

Pietrelli, A., Matkovic, L., Vacotto, M., Lopez-Costa, J. J., Basso, N., and Brusco, A. (2018). Aerobic exercise upregulates the BDNF-serotonin systems and improves the cognitive function in rats. Neurobiol. Learn. Mem. doi: 10. 1016/j.nlm.2018.05.007 [Epub ahead of print].

Pius-Sadowska, E., and Machaliński, B. (2017). BDNF - A key player in cardiovascular system. J. Mol. Cell. Cardiol. 110, 54-60. doi: 10.1016/j.yjmcc. 2017.07.007

Ploutz-Snyder, L. L., and Giamis, E. L. (2001). Orientation and Familiarization to 1RM strength testing in Old and Young women. J. Strength Cond. Res. 15, 519-523.

Rasmussen, P., Brassard, P., Adser, H., Pedersen, M. V., Leick, L., Hart, E., et al. (2009). Evidence for a release of brain-derived neurotrophic factor from the brain during exercise. Exp. Physiol. 94, 1062-1069. doi: 10.1113/expphysiol. 2009.048512
Rojas, V. S., Knicker, A., Hollmann, W., Bloch, W., and Strüder, H. K. (2010). Effect of resistance exercise on serum levels of growth factors in humans. Horm. Metab. Res. 42, 982-986. doi: 10.1055/s-0030-1267950

Rojas Vega, S., Struder, H. K., Vera Wahrmann, B., Schmidt, A., Bloch, W., and Hollmann, W. (2006). Acute BDNF and cortisol response to low-intensity exercise and following ramp incremental exercise to exhaustion in humans. Brain Res. 1121, 59-65. doi: 10.1016/j.brainres.2006.08.105

Sakr, H. F., Abbas, A. M., and El Samanoudy, A. (2015). Effect of vitamin E on cerebral cortical oxidative stress and brain-derived neurotrophic factor gene expression induced by hypoxia and exercise in rats. J. Physiol. Pharmacol. 66, 191-202.

Schiffer, T., Schulte, S., Sperlich, B., Achtzehn, S., Fricke, H., and Strüder H. K. (2011). Lactate infusion at rest increases BDNF blood concentration in humans. Neurosci. Lett. 488, 234-237. doi: 10.1016/j.neulet.2010.11.035

Seifert, T., Brassard, P., Wissenberg, M., Rasmussen, P., Nordby, P., Stallknecht, B., et al. (2010). Endurance training enhances BDNF release from the human brain. Am. J. Physiol. Regul. Integr. Comp. Physiol. 298, R372-R377. doi: 10.1152/ ajpregu.00525.2009

Szuhany, K. L., Bugatti, M., and Otto, M. W. (2015). A meta-analytic review of the effects of exercise on brain-derived neurotrophic factor. J. Psychiatr. Res. 60, 56-64. doi: 10.1016/j.jpsychires.2014.10.003

Tharmaratnam, T., Tabobondung, T., Tabobondung, T., and Doherty, S. (2018). Synergistic effects of brain-derived neurotrophic factor (BDNF) and exercise intensity on memory in the adolescent brain: a commentary. Environ. Health Prev. Med. 23:12. doi: 10.1186/s12199-018-0701-8

von Bohlen Und Halbach, O., and von Bohlen Und Halbach, V. (2018). BDNF effects on dendritic spine morphology and hippocampal function. Cell Tissue Res. doi: 10.1007/s00441-017-2782-x [Epub ahead of print].

Walsh, J. J., Scribbans, T. D., Bentley, R. F., Kellawan, J. M., Gurd, B., and Tschakovsky, M. E. (2016). Neurotrophic growth factor responses to lower body resistance training in older adults. Appl. Physiol. Nutr. Metab. 41, 315-323. doi: 10.1139/apnm-0410

Wrann, C. D., White, J. P., Salogiannnis, J., Laznik-Bogoslavski, D., Wu, J., Ma, D., et al. (2013). Exercise induces hippocampal BDNF through a PGC-1 $1 \alpha /$ FNDC5 pathway. Cell Metab. 18, 649-659. doi: 10.1016/j.cmet.2013.09.008

Xu, B. (2013). BDNF (I)rising from exercise. Cell Metab. 18, 612-614. doi: 10.1016/ j.cmet. 10.008

Yamamoto, M., Sobue, G., Yamamoto, K., Terao, S., and Mitsuma, T. (1996). Expression of mRNAs for neurotrophic factors (NGF, BDNF, NT-3, and GDNF) and their receptors (p75NGFR, trkA, trkB, and trkC) in the adult human peripheral nervous system and nonneural tissues. Neurochem. Res. 21, 929-938. doi: $10.1007 / \mathrm{BF} 02532343$

Yarrow, J. F., White, L. J., McCoy, S. C., and Borst, S. E. (2010). Training augments resistance exercise-induced elevation of circulating brain-derived neurotrophic factor (BDNF). Neurosci. Lett. 479, 161-165. doi: 10.1016/j.neulet.2010. 05.058

Zafra, F., Hengerer, B., Leibrock, J., Thoenen, H., and Lindholm, D. (1990). Activity-dependent regulation of BDNF and NGF mRNAs in the rat hippocampus is mediated by non-NMDA glutamate receptors. EMBO J. 9, 3545-3550.

Zoladz, J. A., Pilc, A., Majerczak, J., Grandys, M., Zapart- Bukowska, J., and Duda, K. (2008). Endurance training increases plasma brain-derived neurotrophic factor concentration in Young healthy men. J. Physiol. Pharmacol. 59(Suppl. 7), 119-132.

Conflict of Interest Statement: The authors declare that the research was conducted in the absence of any commercial or financial relationships that could be construed as a potential conflict of interest.

Copyright (๑) 2018 Pereira, Krause Neto, Calefi, Georgetti, Guerreiro, Zocoler and Gama. This is an open-access article distributed under the terms of the Creative Commons Attribution License (CC BY). The use, distribution or reproduction in other forums is permitted, provided the original author(s) and the copyright owner(s) are credited and that the original publication in this journal is cited, in accordance with accepted academic practice. No use, distribution or reproduction is permitted which does not comply with these terms. 